\title{
Fuzzy Identification and Control Compensation of Unmanned Surface Vehicle Steering System
}

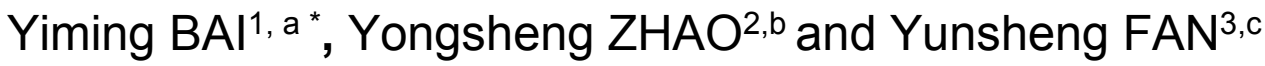 \\ ${ }^{1,2,3}$ Information Science and Technology College, Dalian Maritime University, Dalian 116026, China \\ ahs_bym@126.com, bdmuzys@163.com, c fan_yunsheng@163.com
}

Keywords: Control Compensation; Data Mining; USV Steering System; Fuzzy Modeling.

\begin{abstract}
The steering system modeling and control compensation are the core technologies of autonomous Unmanned Surface Vehicle (USV). The steering characteristics of USV often have strong non-linearity and uncertainty under complex flow, wind and wave. Parameterized math approaches are difficult to achieve satisfactory performance in USV steering system modeling. In this paper, the USV steering system modeling is designed based on data mining technology and fuzzy inference system. This fuzzy data driven method can build the nonlinear function between the heading angle and the nonlinear term in USV steering system. Then, the identification result is applied to the control compensation design for the USV steering system. In the simulation study, a typical USV steering trial is simulated. Compared with the traditional PID control method, the proposed control compensation method demonstrates its rapidity and effectiveness in the control of nonlinear and uncertain USV steering system.
\end{abstract}

\section{Introduction}

Unmanned Surface Vehicle (USV) is one of intelligent motion platforms, which can navigate safely in the real marine environment and complete various tasks. Recently, more and more attention has been paid on USV with advantages of small volume, good flexibility, high speed and no casualties. However, USV steering characteristics are often affected by the complex flow, wind and wave [1]. It is difficult to generate an accurate model of USV steering system. Furthermore, this inherent non-linearity and uncertainty of USV steering system make it difficult to control using the traditional PID control methods. Efficient control systems are very important to improve the observation effect of reconnaissance equipment and the precision of the weaponry system [2]. The USV control system needs to strengthen its control ability for highly dynamic and unpredictable marine environment [3]. Therefore advanced control strategies are kind of need to get satisfactory maneuvering performance.

In this paper, USV steering system model is generated with data mining technology and fuzzy inference system. Then a USV steering compensation controller is designed based on this model. In the USV steering modeling, the method based on fuzzy inference system is a data driven method. The input and output sampling recordings of USV trials are used to construct nonlinear part in USV steering system directly regardless of the internal mechanism and hydrodynamic factors [4]. In the USV steering control, the identification result is applied to the design of USV steering PD control compensation, which can weaken the non-linearity and uncertainty to achieve better control performance [5]. Compared with the traditional PID steering control method, simulation results validate the rapidity and effectiveness of this control compensation method.

\section{Fuzzy modeling method for USV steering system}

Recently, fuzzy modeling techniques have been successfully applied to modeling complex systems. This section proposes a data mining algorithm to extract a fuzzy rule base for non-linear USV steering system modeling. To identify the steering model of USV with fuzzy inference systems, it needs to collect enough experimental data and then employ the data mining algorithm to extract 
fuzzy rules for non-linear USV steering system modeling. In this paper, the data records are collected from actual trials of the Lanxin Ship of Dalian Maritime University of China in fig.1.

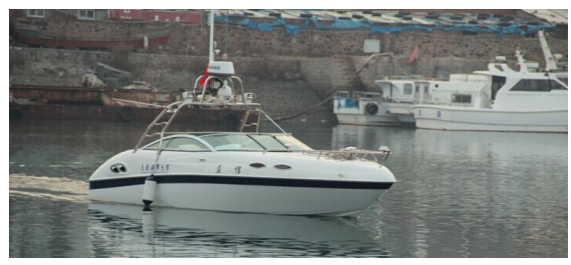

Fig.1 the Lanxin Ship

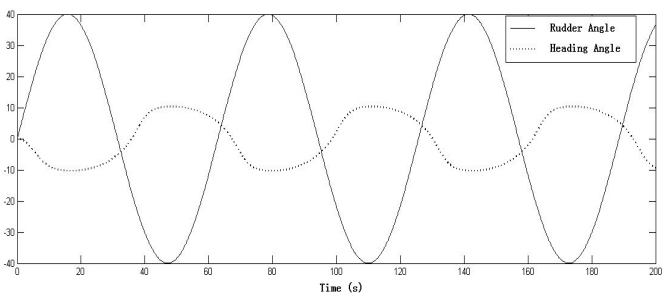

Fig. 2 the relationship between the rudder angle $\delta$ and the heading angle $\psi$

A set of rudder angle and heading angle variation data pairs $\left(\delta^{(p)}, \dot{\psi}^{(p)}\right)$ is obtained, where $\mathrm{p}=1,2, \ldots \mathrm{N}, \mathrm{N}$ is the total number of data pairs. The fig. 2 depicts the collected data relationship between the rudder angle and the heading angle of a typical trial. The task is to generate a set of fuzzy rules from the given input-output pairs to model the nonlinear USV steering system.

The rule base can be built as the following five steps:

Step 1 Partition the input and output spaces

Assume that the domain intervals of $\delta$ and $\dot{\psi}$ are $\left[\delta^{-}, \delta^{+}\right]$and $\left[\dot{\psi}^{-}, \dot{\psi}^{+}\right]$, respectively. Each interval is divided into $d_{i}(i=1,2)$ regions by triangular membership functions. $d_{i}$ may be different for different variable, and the lengths of these regions can be equal or unequal. Fig. 3 shows an example that the domain interval is divided into five equal regions.

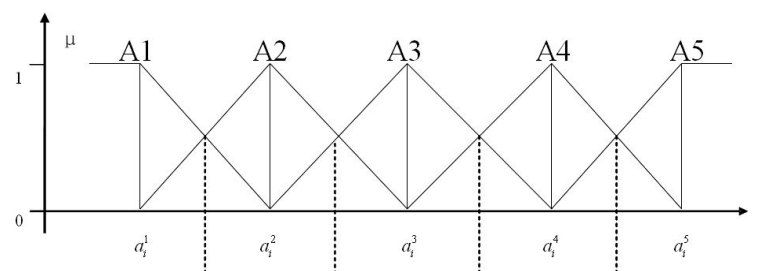

Fig. 3 Equal partitioning membership function

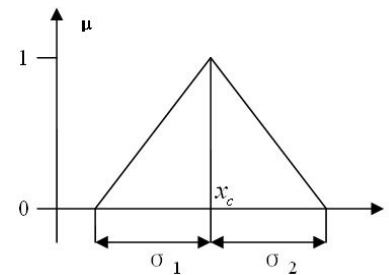

Fig. 4 Triangular membership function

Step 2 Transform numerical data-pairs into fuzzy data-pairs

$\mathrm{A}_{\mathrm{k}}(\mathrm{k}=1,2, \ldots 5)$ is the linguistic label associated with the fuzzy membership function. Then the numerical data can be characterized by the triangular membership function in fig. 4 defined as:

$$
\begin{aligned}
& \mu\left(x ; x_{c}, \sigma_{1}\right)=\frac{\sigma_{1}-\left(x_{c}-x\right)}{\sigma}, x \in\left[x_{c}-\sigma_{1}, x_{c}\right] \\
& \mu\left(x ; x_{c}, \sigma_{2}\right)=\frac{\sigma_{2}-\left(x-x_{c}\right)}{\sigma_{2}}, x \in\left[x_{c}, x_{c}+\sigma_{2}\right] \\
& \mu\left(x ; x_{c}, \sigma_{1}, \sigma_{2}\right)=0, x \in R-\left[x_{c}-\sigma_{1}, x_{c}+\sigma_{2}\right]
\end{aligned}
$$

where $\sigma_{1}, \sigma_{2}$ and xc are determined by the partition in step 1. Then a numerical data-pair: $D_{p}=\left(\delta^{(p)} ; \dot{\psi}^{(p)}\right),(p=1,2, \ldots, N)$

could be converted into a fuzzy data-pair:

$F_{p}=\left(\mu\left(\delta^{(p)}\right) ; \mu\left(\dot{\psi}^{(p)}\right)\right),(p=1,2, \ldots, N)$.

Step 3 Generating a fuzzy rule base on support degree

The degree of support in Data Mining concept is utilized in fuzzy rule generation. The definition of support is to tell whether a fuzzy rule is strong enough to summarize the data information over the region covered by this rule. The support degree of rule $\mathrm{i}$ is calculated as follow:

$$
\sup _{B^{\prime}}(R i)=\frac{\sum_{p=1}^{N} \mu_{\left(\delta^{p}\right)}^{A^{h^{1}}} \mu_{\left(\dot{\psi}^{p}\right)}^{B^{B^{2}}}}{\sum_{p=1}^{N} \mu_{\left(\delta^{p}\right)}^{A^{1^{1}}}},
$$

where $\mathrm{N}$ is the total number of data pairs, $l_{1}$ and $l_{2}$ are fixed according to the input and output spaces partition. The linguistic label $\mathrm{B}_{\mathrm{k}}$ is chosen as the fuzzy rule's THEN part, whose $\sup _{B^{k}}(R i)(\mathrm{k}$ 
$\left.\in\left[1,2, \ldots, 1_{2}\right]\right)$ is the maximum value among the $1_{2}$ values for rule $i$. The commonly used flow chart of fuzzy rule generation is shown in fig.5.

Step 4 Reduce the rule number

A complete rule base is generated from the above three steps. In some case, few data pairs may be covered in some of the input partition. And the degree of support for these rules is very weak. Then, the trivial rules need to be removed from the rule base.

Step 5 The centroid defuzzification

The following centroid defuzzification algorithm is used to determine the output $\dot{\psi}$ for given input $\delta$ :

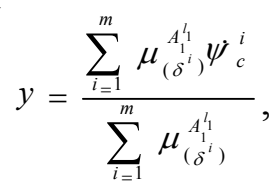

where $\mathrm{m}$ is the total number of fuzzy rules, $\dot{\psi}_{c}^{i}$ denotes the center value of output region $\mathrm{i}$.

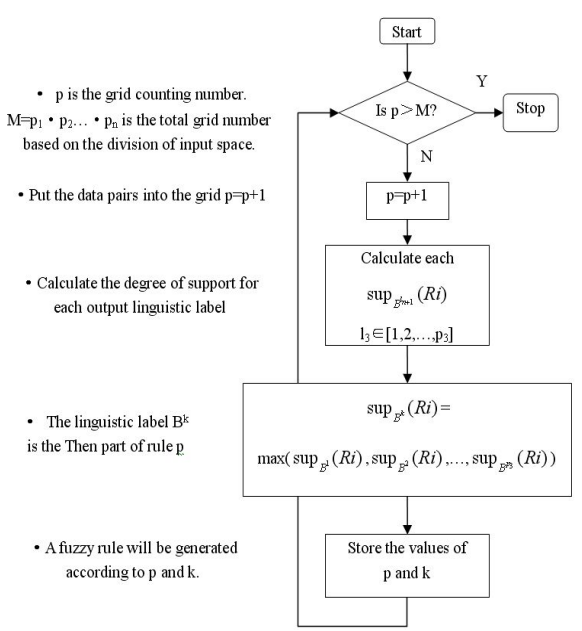

Fig. 5 The flow chart of fuzzy rule generation

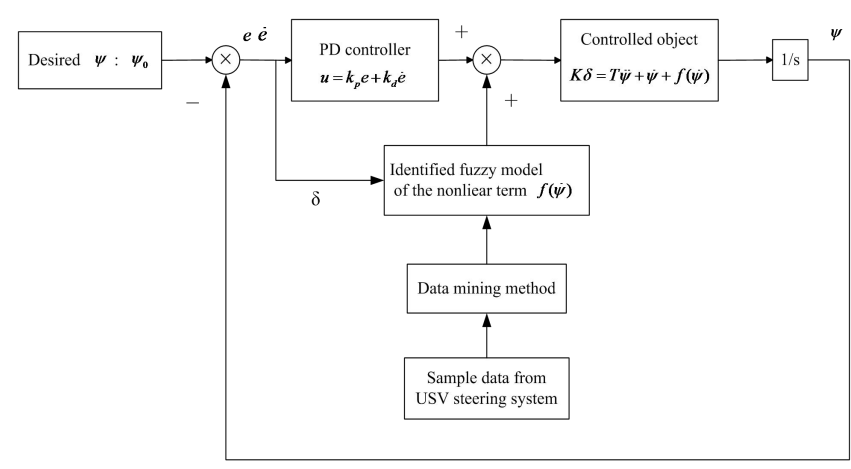

Fig.6 Schematic diagram of control compensation

Thus, a fuzzy model can be built by the above five steps. This fuzzy model can reflect the nonlinear relationship in USV steering system for the design of control compensation.

\section{The design of control compensation for USV steering system}

Considering the non-linearity and uncertainty, USV steering system can be approximated as a Nomoto model:

$$
K \delta=T \ddot{\psi}+\dot{\psi}+f(\dot{\psi})
$$

$\delta$ is the rudder angle, $\psi$ is the heading angle, $\mathrm{T}$ and $\mathrm{K}$ are steering indexes, $f(\dot{\psi})$ is the nonlinear part of the USV steering system. It is a mature method to identify the $\mathrm{K}$, $\mathrm{T}$ steering indexes by calculating the main characteristic parameters of USV [8].

The nonlinear part of USV steering system can be approximated as the function $f(\dot{\psi})$ by reducing dimension of the USV steering model and ignoring some dynamic characteristics of the USV steering system. At present, it is very difficult to identify the nonlinear term $f(\dot{\psi})$ of USV steering system with explicit math model because of the uncertainty disturbance of complex flow, wind and wave. And in controlling the approximated control object $T \ddot{\psi}+\dot{\psi}+f(\dot{\psi})$, traditional PID controller has the limitation in control the nonlinear part $f(\dot{\psi})$.

In this paper, a control compensation method based on fuzzy identification of nonlinear term is proposed. Fig. 6 depicts the flow chart of the overall control systems. The PD controller is designed for the linear part $T \ddot{\psi}+\dot{\psi}$ and the fuzzy identification of $f(\dot{\psi})$ is considered as the control compensation for the USV steering system. Thus, the feedback parameter of compensation can be adjusted to improve the performance of the PD controller design for the USV steering system. 
A control system of USV steering is simulated to verify this control compensation theory. The controlled object is approximated as a Nomoto model. And the parameters of Nomoto model can be identified with the sample data from various USV steering trials [9]. And the simulation results of the tracking errors are shown in fig.7. The results demonstrate that the PD control compensation method has its advantage, such as the shorter tracking time and the smaller overshoot than PID controller.

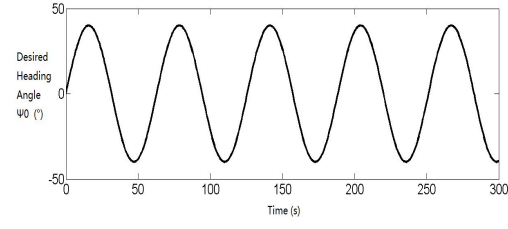

7.1 The desired heading angle

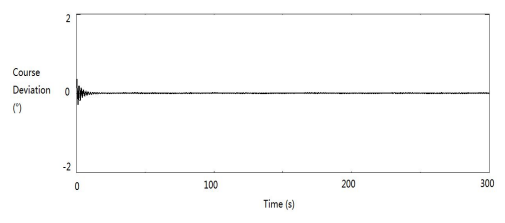

7.2 The course tracking deviation of PD control compensation

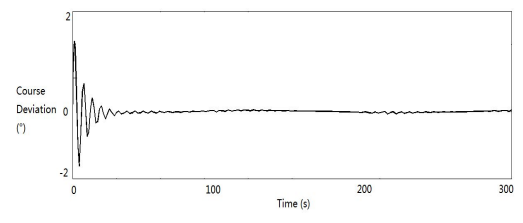

7.3 The course tracking deviation of PID controller

Fig. 7 The course tracking results

\section{Acknowledgement}

This work is supported by the Fundamental Research Funds for the Central Universities 2015.

\section{Conclusion}

This paper develops a framework of modeling USV steering system by the use of data mining techniques. And then employs this USV steering model in the design of control compensation. For the nonlinear identification capacity of fuzzy modeling, simulation results demonstrate that the proposed data-driven approaches for modeling and compensation control technique are effective and useful in the improvement of control performance.

\section{References}

[1] Caccia M, Bibuli M, Bono R, et al. Basic navigation, guidance and control of an unmanned surface vehicle[J]. Autonomous Robots, 2008, 25(4): 349-365.

[2] Craven P J, Sutton R, Burns R S. Control strategies for unmanned underwater vehicles[J]. Journal of Navigation, 1998, 51(01): 79-105.

[3] Kurnaz S, Cetin O, Kaynak O. Fuzzy logic based approach to design of flight control and navigation tasks for autonomous unmanned aerial vehicles[J]. Journal of Intelligent and Robotic Systems, 2009, 54(1-3): 229-244.

[4] Wang L X. The WM method completed: a flexible fuzzy system approach to data $\operatorname{mining}[\mathrm{J}]$. Fuzzy Systems, IEEE Transactions on, 2003, 11(6): 768-782.

[5] Wang Y, Wang D, Chai T. Extraction and adaptation of fuzzy rules for friction modeling and control compensation[J]. Fuzzy Systems, IEEE Transactions on, 2011, 19(4): 682-693.

[6] Bai Y, Li T, Gao X. Ship maneuvering modeling based on fuzzy rules extraction and optimization[M]//Advances in Neural Networks-ISNN 2013. Springer Berlin Heidelberg, 2013: 429-435.

[7] Wang Y F, Wang D, Chai T Y. Extraction of fuzzy rules with completeness and robustness[J]. 2010.

[8] Bhattacharyya S K, Haddara M R. Parametric identification for nonlinear ship maneuvering[J]. Journal of ship research, 2006, 50(3): 197-207.

[9] Luo W L. On the Modeling of Ship Maneuvering Motion by Using Support Vector Machines[D]. $\mathrm{Ph}$. D. thesis, Shanghai Jiao Tong University, Shanghai, China.(in Chinese), 2009. 